\title{
Three-dimensional computed tomography angiography and bronchography combined with three-dimensional printing for thoracoscopic pulmonary segmentectomy in stage IA non-small cell lung cancer
}

\author{
Wenbin $\mathrm{Hu}^{1}$, Kang Zhang ${ }^{1}$, Xiaoliang Han ${ }^{1}$, Jiaming Zhao ${ }^{1}$, Guzong Wang ${ }^{1}$, Shunda Yuan ${ }^{1}$, Binjun $\mathrm{He}^{2}$ \\ ${ }^{1}$ Department of Cardiothoracic Surgery, Affiliated Hospital of Shaoxing University (The Shaoxing Municipal Hospital), Shaoxing, China; \\ ${ }^{2}$ Department of Thoracosurgery, Shaoxing People's Hospital, Zhejiang University School of Medicine, Shaoxing, China \\ Contributions: (I) Conception and design: W Hu, B He, S Yuan; (II) Administrative support: K Zhang, J Zhao; (III) Provision of study materials or \\ patients: G Wang, X Hang; (IV) Collection and assembly of data: W Hu; (V) Data analysis and interpretation: All authors; (VI) Manuscript writing: \\ All authors; (VII) Final approval of manuscript: All authors. \\ Correspondence to: Binjun He. Department of Thoracosurgery, Shaoxing People's Hospital, Zhejiang University School of Medicine, Shaoxing 312000 , \\ China. Email: hebinjun@sina.cn.
}

Background: Compared with lobectomy, the anatomical structure of the lung segment is relatively complex and easy to occur variation, thus it increases the difficulty and risk of precise segmentectomy. The application of three-dimensional computed tomography bronchography and angiography (3D-CTBA) combined with a three-dimensional printing (3D printing) model can ensure the safety of operation and simplify the surgical procedure to a certain extent. We aimed to estimate the value of 3D-CTBA and 3D printing in thoracoscopic precise pulmonary segmentectomy.

Methods: We retrospectively reviewed the clinical data of 65 patients who underwent anatomical segmentectomy at the Affiliated Hospital of Shaoxing University from January 2019 to August 2020. The patients were divided into two groups: a 3D-CTBA combined with $3 \mathrm{D}$ printing group (30 patients) and a general group (35 patients). The perioperative data of the two groups were compared.

Results: Compared with the general segmentectomy group at the same period in our center, the surgery time of the group guided by 3D-CTBA and 3D printing was significantly shorter. Intraoperative blood loss in the 3D-CTBA and 3D printing group was also apparently lower than in the general group. Hospital stay and postoperative chest tube duration showed no significant differences between the two groups, and neither did postoperative complications such as pneumonia, hemoptysis, arrhythmia, and pulmonary air leakage.

Conclusions: 3D-CTBA combined with 3D printing clearly identifies the precise pulmonary segmental structures, avoids intraoperative accidental injury, reduces intraoperative blood loss, shortens the operation time and improves the safety of thoracoscopic pulmonary segmentectomy in stage IA non-small cell lung cancer (NSCLC).

Keywords: Three-dimensional computed tomography bronchography and angiography (3D-CTBA); threedimensional printing (3D printing); pulmonary segmentectomy; lung cancer

Submitted Nov 21, 2020. Accepted for publication Feb 04, 2021.

doi: $10.21037 /$ jtd-21-16

View this article at: http://dx.doi.org/10.21037/jtd-21-16 


\section{Introduction}

With the broad application of low-dose chest computed tomography in routine physical examinations, early stage lung cancer is increasingly easier to detect. In recent years, growing evidence suggests that pulmonary segmentectomy has the same oncological outcomes as pulmonary lobectomy in stage IA non-small cell lung cancer (NSCLC). Anatomic segmentectomy can minimally resect the lesion, maximally remain healthy lung tissue, and avoid unnecessary loss of lung function. Compared with wedge resection, segmentectomy ensures sufficient surgical margins and lymph node sampling for accurate tumor staging. Therefore, intentional segmentectomy has gradually become the first choice for stage IA NSCLC (1-3). Compromise segmentectomy is also the best treatment option for patients with poor lung function who cannot tolerate lobectomy.

Compared with lobectomy, the anatomical structure of the plumonary segment is relatively complex and easy to occur variation. It is difficult to accurately display the meticulous anatomical structure of the pulmonary artery, vein, and bronchus based on preoperative twodimensional CT images, thus it increases the difficulty and risk of precise segmentectomy. Therefore, it is particularly important to find a technique that better displays the precise anatomical structure in order to guide the resection of the pulmonary segment. Previous studies mostly focused on simple three-dimensional reconstruction technology. In our center, we use three-dimensional computed tomography bronchography and angiography (3D-CTBA) which can greatly improve the discernibility of CT images, combined with three-dimensional printing (3D printing) can accurately, stereoscopically, and multi-dimensionally present the anatomical structure of the target pulmonary artery and vein, bronchus lesion, and surrounding tissue. This method can provide a better sense of space and tactile feedback, achieve accurate matching between the virtual reconstruction model and the actual anatomical structure and guide the precise implementation of segmentectomy.

In this paper, we retrospectively collected and analyzed the detaled clinical features of patients who underwent segmentectomy in our center and evaluated the utility of 3D-CTBA combined with $3 \mathrm{D}$ printing for anatomical pulmonary segmentectomy in the treatment of stage IA NSCLC. We present the following article in accordance with the MDAR reporting checklist (available at http:// dx.doi.org/10.21037/jtd-21-16).

\section{Methods}

\section{Patient selection}

In this retrospective study, we collected and analyzed the detailed medical records of 65 patients who underwent anatomical segmentectomy at the Affiliated Hospital of Shaoxing University between January 2019 and August 2020. We applied 3D-CTBA combined with 3D printing technology to transform 2D CT images into 3D printing models for preoperative planning in 30 patients, while the preoperative plan for the remaining 35 patients was undertaken with the standard two-dimensional enhanced chest CT. Accordingly, we divided the 65 patients into two groups: the 3D-CTBA combined with $3 \mathrm{D}$ printing group and the general group. The perioperative data of the two groups were compared. According to the National Comprehensive Cancer Network (NCCN) guidelines, the recommended operative indications for pulmonary segmentectomy in our center are as follows: (I) clinical diagnosis of stage IA NSCLC nodules with diameter $\leq 2 \mathrm{~cm}$; (II) CT showing that the nodule has $\geq 50 \%$ groundglass appearance, and the nodule conforms a long doubling time by radiologic surveillance ( $\geq 400$ days); (III) the nodule is deeply positioned, difficult to locate, and close to the adjacent vessels; (IV) a suspected solitary lung metastatic tumor deep in the lung that is not feasible by wedge resection; (V) patients with poor pulmonary function or other serious complications who cannot tolerate lobectomy.

All procedures performed in this study involving human participants were in accordance with the Declaration of Helsinki (as revised in 2013). The study was approved by the Institutional Review Board of the Affiliated Hospital of Shaoxing University, and written informed consent was obtained from patients prior to surgery.

\section{Preoperative planning of the pulmonary segmentectomy guided by $3 D-C T B A$ and $3 D$ printing}

\section{Obtaining high quality CT images}

In our institution, all patients underwent 3D-CTBA scanning with a high-speed 64-channel multi-detector CT scanner (SOMATOM Definition AS, Siemens, Germany). Vascular access was obtained in the median vein of the right elbow. The scan ranged from the thoracic entrance to the diaphragm level. An automatic injector for vessel enhancement was used to inject $40 \mathrm{~mL}$ of iohexol at a flow rate of $5.0 \mathrm{~mL} / \mathrm{s}$. After the contrast medium injection, 

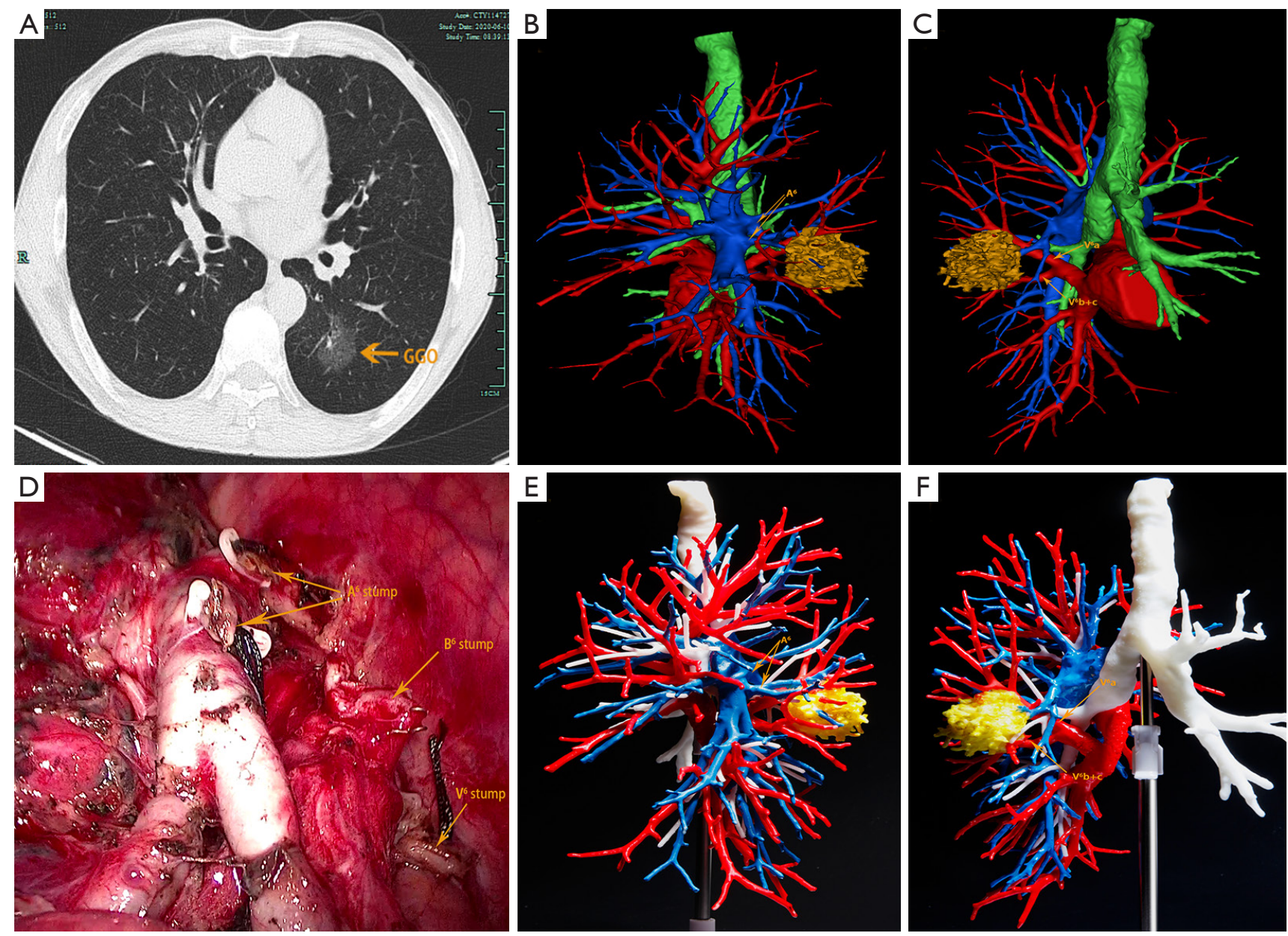

Figure 1 The CT scan, 3D-CTBA, and 3D model of a 56-year-old female patient. One GGO is found in the left lower lobe. According to the scrutiny of the 3D simulation and 3D model, anatomical segmentectomy with left S6 is performed. (A) The CT scan shows that a GGO is located in the left lobe $\left(\mathrm{S}^{6}\right)$; (B) the reconstructed image shows the relationship between the lesion and arteries; (C) 3D reconstruction imaging shows the location relationship between the nodule and the pulmonary vessels; (D) the surgical wound after segmentectomy; (E,F) the $3 \mathrm{D}$ printed model. 3D-CTBA, three-dimensional computed tomography bronchography and angiography; GGO, ground-glass opacity.

$30 \mathrm{~mL}$ of normal saline was immediately injected at the same flow rate. Axial thin-section CT images of the whole lung were reconstructed using a slice thickness of $0.7 \mathrm{~mm}$.

\section{The 3D-CTAB reconstruction}

The thin-slice two-dimensional CT images in DICOM format were exported from PACS and transferred to Mimics software (Materialise, Belgium). The nodules, the trachea and the pulmonary vessels were reconstructed and color-coded by the different CT values. Then, three-dimensional reconstruction images and two-dimensional CT images were carefully compared to determine whether there were anatomical variations and deformities. Finally, the location of the lesion was accurately identified, and the attribution of the lung segment and subpulmonary segment was defined (Figures 1 and 2).

\section{D printing}

The 3D-CTBA imaging reconstructed by Mimics software was exported and saved as an STL file mode. The STL format file of the 3D digital models was imported into the preprocessing software (Materialise Magics) and was checked, repaired and typeset programmed. Multilayer virtual slices were formed by "layering". Each slice corresponded to a real printing layer of the 3D model, and the file form recognized by the $3 \mathrm{D}$ printer was generated. Next, the printing parameters, such as layer thickness, printing speed, and filling density were set according to the requirements. Then the model layer thickness, printing speed, 

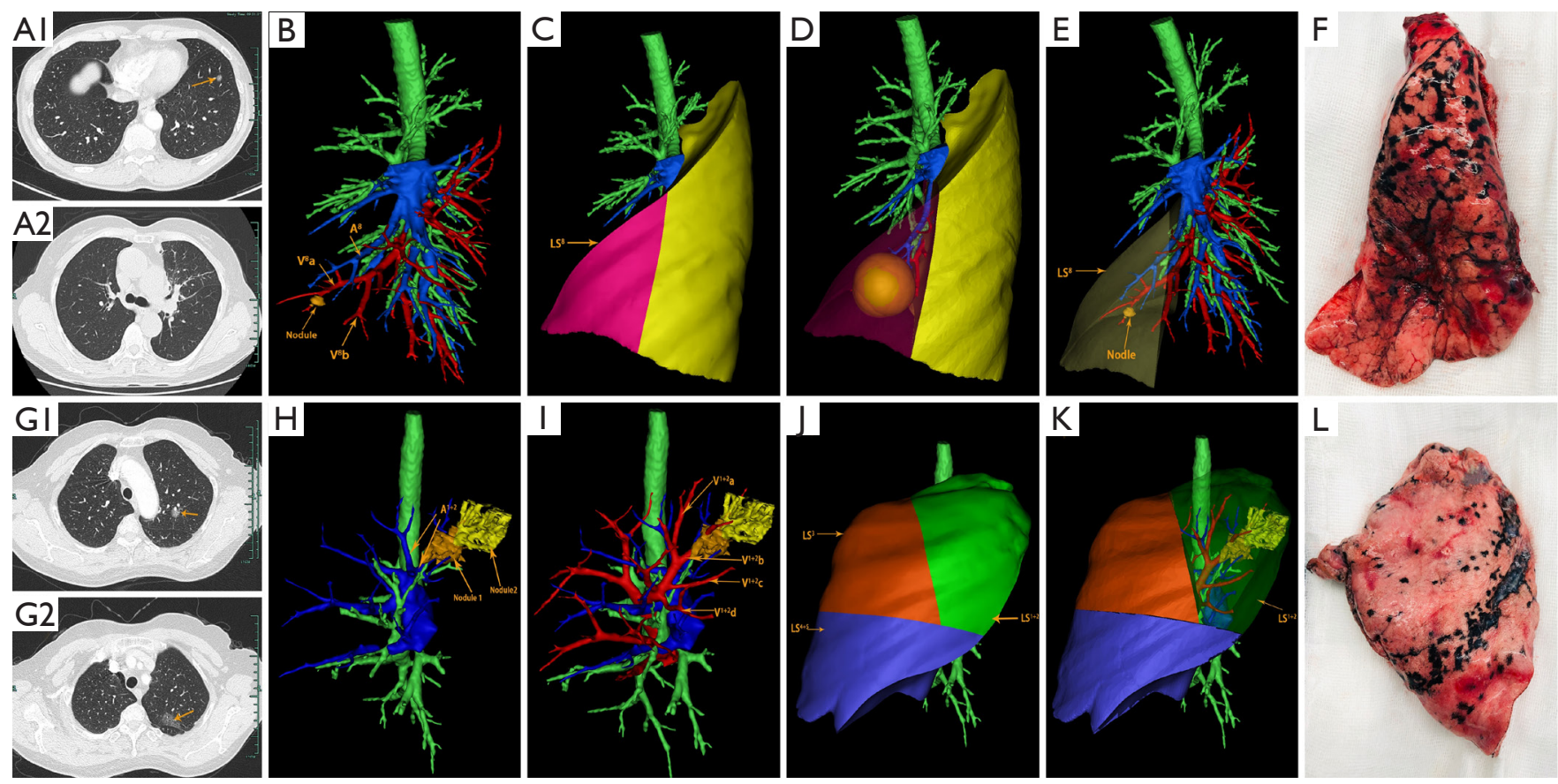

Figure 2 The CT scan and 3D-CTBA resected target lung segment of two patients. (A1) The CT scan shows that a ground glass nodule is located in the left lower lobe $\left(\mathrm{S}^{8}\right)$ of one patient; (A2) the CT scan shows no atelectasis after surgery; (B) the reconstructed image shows the relationship between the nodule and pulmonary vessels; $(\mathrm{C})$ the planned resection region $\left(\mathrm{LS}^{8}\right)$; (D) the evaluation to ensure the surgical margin; (E) the target lung segment to be resected; (F) the surgical specimens of the resected lung segment; (G1,G2) CT scans show that two ground glass nodules were located in the left upper lobe $\left(\mathrm{S}^{1+2}\right)$ of another patient; $(\mathrm{H}) 3 \mathrm{D}$ reconstruction imaging shows the location relationship between the nodules and pulmonary arteries (I) 3D reconstruction imaging shows the location relationship between the nodules and pulmonary veins; $(\mathrm{J}, \mathrm{K})$ the planned resection region and the target lung segment; $(\mathrm{L})$ the surgical specimens of the resected lung segment. 3D-CTBA, three-dimensional computed tomography bronchography and angiography.

filling density, and other printing parameters were set to complete the accurate printing of the model (Figure 1E,F).

\section{Surgical procedures}

According to the dynamic 3D-CTBA imaging and 3D printing model, the structure of the targeted segmental blood vessels and bronchi needing to be excised were analyzed, the anatomic variation was identified, and the surgical procedure planned. Patients were administered general anesthesia with double lumen tracheal intubation. All patients underwent thoracoscopic segmentectomy through two hole approach. During surgery, the $3 \mathrm{D}$ printing model was placed beside the monitor, and the actual anatomical structures were matched with the virtual reconstructed structures. The real-time and multidimensional 3D printing model guided the accurate dissection of the targeted bronchi and vessels one by one.

We use a modified "inflation-deflation" technique to ascertain the segmental and subsegmental borders. After the targeted pulmonary vessels and bronchi were dissected, pure oxygen bipulmonary ventilation was initiated, and then one-lung ventilation was maintained on the non-operative side lung. Approximately 15 minutes later, the targeted segments or subsegments were kept expanded but the preserved segments were completely deflated. Therefore, the intersegmental border was clearly discerned. The interface between the inflation and deflation was separated along the intersegmental veins to the outer one third of the pulmonary parenchyma by using an ultrasonic scalpel. The residual pulmonary parenchyma was resected by staplers.

The surgical margins of all malignant nodules were required to be $\geq 2 \mathrm{~cm}$ or at least larger than the tumor diameter (Figure 2D). If necessary, multisegmental resection was performed. Frozen section examination was performed during operation in all specimens. N1 and N2 lymph nodes were also routinely sampled and sent for frozen section pathology during surgery 
Table 1 Clinical characteristics of each group

\begin{tabular}{|c|c|c|}
\hline Variable & 3D-CTBA and 3D printing group $(n=30)$ & General group $(n=35)$ \\
\hline \multicolumn{3}{|l|}{ Age, years } \\
\hline Mean (SD) & $61.3(9.5)$ & $62.7(9.4)$ \\
\hline Range & $37-77$ & $35-79$ \\
\hline \multicolumn{3}{|l|}{ Sex } \\
\hline Male & $14(46.7 \%)$ & $16(47.3 \%)$ \\
\hline Mean nodule size (mm) (SD) & $10.5(3.2)$ & $11.3(4.1)$ \\
\hline \multicolumn{3}{|l|}{ Nodule location } \\
\hline RUL & $10(33.3 \%)$ & $11(27.5 \%)$ \\
\hline \multicolumn{3}{|l|}{ Pathological diagnosis } \\
\hline Benign & $5(13.9 \%)$ & $6(15 \%)$ \\
\hline AAH & $1(2.8 \%)$ & $2(5 \%)$ \\
\hline AIS & $3(8.3 \%)$ & $5(12.5 \%)$ \\
\hline MIA & $23(65.7 \%)$ & $22(55 \%)$ \\
\hline IAC & $4(11.1 \%)$ & $5(12.5 \%)$ \\
\hline
\end{tabular}

Data are presented as $\mathrm{n}(\%)$ and means (SD) unless specified otherwise. 3D-CTBA, three-dimensional computed tomography bronchography and angiography; RUL, right upper lobe; RLL, right lower lobe; LUL, left upper lobe; LLL, left lower lobe; AAH, atypical adenomatous hyperplasia; AIS, adenocarcinoma in situ; MIA, minimally invasive adenocarcinoma; IAC, invasive adenocarcinoma.

in malignant cases. In the case of lymph node involvement, lobectomy and systematic lymphadenectomy was performed.

\section{Statistical analysis}

All collected clinical data were analyzed by SPSS 21.0 software (IBM Corp., Armonk, NY, USA). The continuous variables were presented as mean \pm standard deviation $(\mathrm{SD})$ and number (\%) for categorical variables. Between groups, continuous variables were analyzed using the $t$-test, and categorical variables using the $\chi^{2}$ or Fisher's exact test. A P value of $<0.05$ was considered statistically significant.

\section{Results}

\section{Clinical characteristics of the patients}

The clinical characteristics of the two patient groups are summarized in Table 1. Ultimately, 30 patients were included in the $3 \mathrm{D}-\mathrm{CTBA}$ and $3 \mathrm{D}$ printing group. The group included 16 women (53.3\%) and 14 men (46.7\%) and had a mean age of 61.3 (9.5) years. The 30 patients had a total of 36 nodules, and the mean diameter of the nodules was $10.5(3.2) \mathrm{mm}$. The nodules were located in the right upper lobe in 10 cases, right lower lobe in three cases, left upper lobe in 14 cases, and the left lower lobe in three cases. The postoperative pathological diagnoses included: inflammatory pseudotumor in three cases, tuberculoma in one case, sclerosing hemangioma in one case, atypical adenomatous hyperplasia (AAH) in one case, adenocarcinoma in situ (AIS) in three cases, minimally invasive adenocarcinoma (MIA) in 22 cases, and invasive adenocarcinoma (IAC) in four cases. There were no significant statistically differences between the two groups.

\section{The accurate nodule location and surgical procedure selection}

The details of the accurate nodule location and 
Table 2 Nodule location and segmentectomy position of the two groups

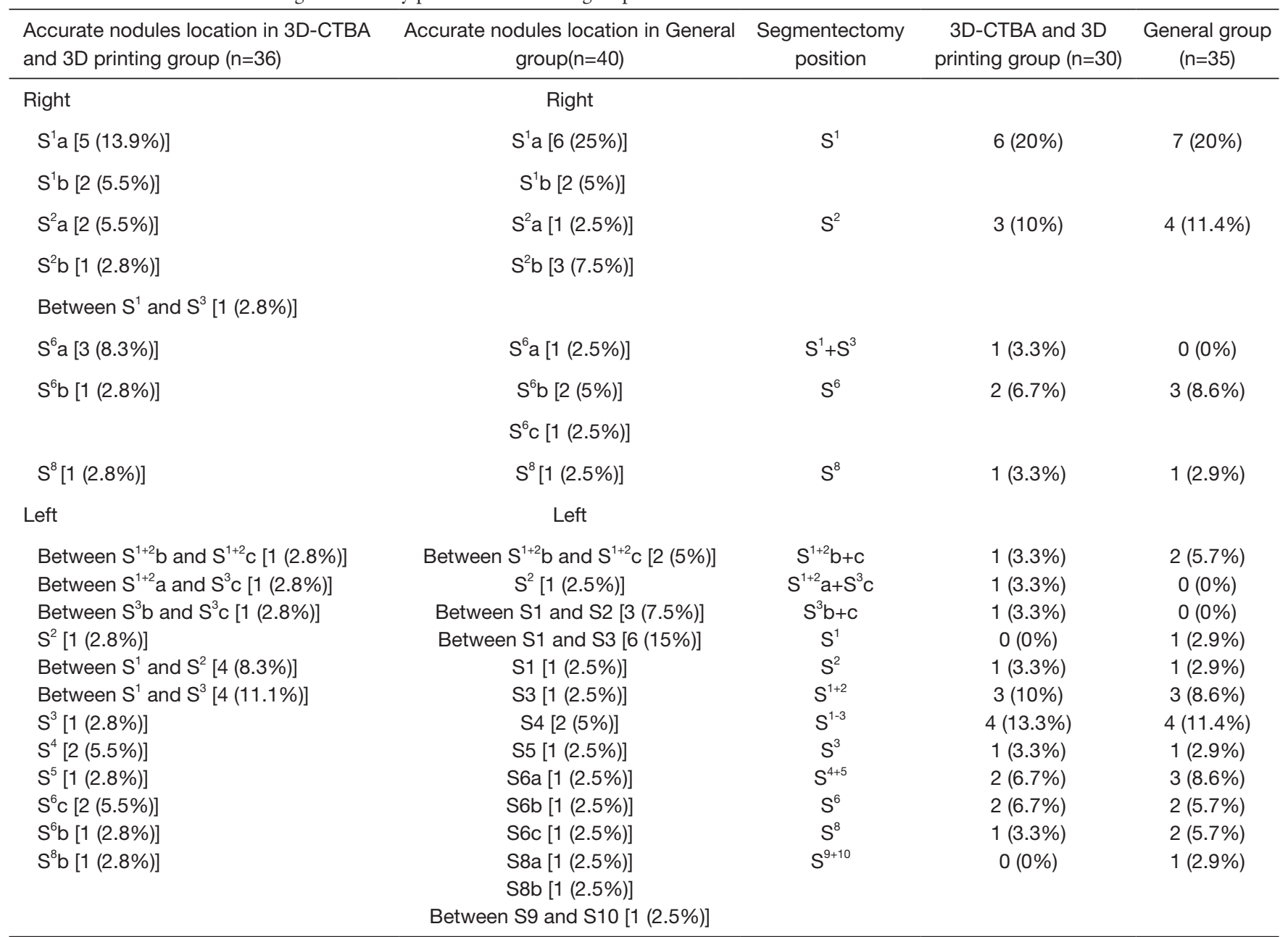

Data are presented as $\mathrm{n}(\%)$ and means (SD) unless specified otherwise. 3D-CTBA, three-dimensional computed tomography bronchography and angiography.

segmentectomy position are presented in Table 2. Nodules were mainly found in the upper lobe, accounting for $86.1 \%$ of the distribution. In the $3 \mathrm{D}-\mathrm{CTBA}$ and $3 \mathrm{D}$ printing group, standard segmental resection was performed in 27 cases, subsegmental resection was performed in two cases, and combined segmental resection was performed in one case. Meanwhile, the general group underwent standard segmental resection in 33 cases, subsegmental resection in two cases and combined segmental resection in one case.

\section{Intraoperative and postoperative data}

Segmentectomy was successfully and accurately completed for all patients. None of the patients were converted to open chest surgery, the frozen sections of lymph nodes were all negative, and no case was changed to lobectomy. The surgical margins of each patient met the margin requirement. Surgery time was shorter, and blood loss was less in the 3D-CTBA and 3D printing group compared with the general segmentectomy group at the same period in our center. There were no significant statistically differences between the two groups in postoperative chest tube duration or hospital stay. Postoperative complications of arrhythmia, pneumonia, pulmonary air leakage and hemoptysis showed no significant statistically differences between the two groups. Relevant results are indicated in Table 3.

\section{Discussion}

Although lobectomy is still recognized as the standard 
Table 3 Intraoperative and postoperative data of the two groups

\begin{tabular}{|c|c|c|c|}
\hline Variables & General group $(n=35)$ & 3D-CTBA and 3D printing group $(n=30)$ & $\mathrm{P}$ \\
\hline Intraoperative blood loss, mL (SD) & $114.3(50.6)$ & $81.3(42.2)$ & 0.024 \\
\hline Postoperative chest tube drainage duration, days & $3.9(1.6)$ & $3.5(1.7)$ & 0.190 \\
\hline Postoperative hospital stays, days & $5.8(1.7)$ & $5.3(1.6)$ & 0.320 \\
\hline Pneumonia & 2 & 1 & 1.000 \\
\hline Hemoptysis & 1 & 0 & 1.000 \\
\hline Arrhythmia & 4 & 3 & 1.000 \\
\hline Pulmonary air leakage & 2 & 1 & 1.000 \\
\hline
\end{tabular}

Data are presented as $\mathrm{n}(\%)$ and means (SD) unless specified otherwise. 3D-CTBA, three-dimensional computed tomography bronchography and angiography.

surgical treatment for lung cancer (4), increasing evidencebased medical outcomes show that in the treatment of stage IA NSCLC, especially when the tumor diameter is less than $2 \mathrm{~cm}$, the long-term efficacy of segmentectomy and lobectomy is comparable. A propensity score matching conclusion from the Surveillance, Epidemiology, and End Results (SEER) database shows that for stage IA IAC, segmentectomy may have equal oncologic outcomes to those of lobectomy (5). Data from the University of Pittsburgh Medical Center show that in the case of cT1cN0M0 NSCLC, there was no significant difference in recurrence-free or overall survival at 5 years between segmentectomy and lobectomy (6). Similarly, a propensity score matching conclusion from the National Cancer Database (NCDB) shows that survival is similar between lobectomy and segmentectomy for clinical T1N0 and unsuspected 89 pathological N1/N2 nodal metastases (7). Therefore, for patients with stage IA peripheral type NSCLC with less than $2 \mathrm{~cm}$ of ground glass nodules, survival after segmentectomy is no worse than that of patients with lobectomy, and this view is now widely recognized by the mainstream academic community. As such, an increasing number of medical institutions currently perform intentional precise pulmonary segmentectomy to treat stage IA NSCLC.

As one of the representative methods of precise surgery, thoracoscopic segmentectomy is based on precise anatomy, can minimally resect the lesion, maximally remain healthy lung tissue, maximally preserve healthy lung tissue to protect the patient's lung function, avoid unnecessary loss of health lung function and improve the quality of the patient's postoperative life (8). However, compared with thoracoscopic lobectomy, thoracoscopic segmentectomy require a greater understanding of the anatomical structure of the lung segment. In the deeper anatomical structures, the relationship between the bronchus and pulmonary arteries and veins becomes more complicated and varied. Especially in complex pulmonary segmental resection, it is difficult for surgeons to recall the $3 \mathrm{D}$ visualization based only on the preoperative 2D CT images. If the precise anatomical structure of the pulmonary segment or subsegment cannot be completely discriminated before surgery, intraoperative vascular injury or false rupture is likely to occur, requiring lobectomy or open chest surgery.

In recent years, a personalized $3 \mathrm{D}-\mathrm{CTBA}$ and $3 \mathrm{D}$ printing model has shown a unique advantage in the preoperative planning of complicated thoracic surgeries such as pulmonary segmentectomy (9-13). Although the need for a 3D-CTBA and 3D printing model in surgical planning of more simple segmental resections may not be necessary, it plays a very important role in complex segmental resection. Preoperative three-dimensional reconstruction can clearly display the precise structures of the pulmonary segmental and subsegment structures. Surgical simulation with threedimensional reconstruction images is also helpful for planning the surgical approach. Preoperative determination of the target pulmonary artery, vein and bronchus can shorten the time of intraoperative identification. The $3 \mathrm{D}$ models can be placed next to the video-assisted thoracoscopic display screen for real-time contrast during surgery where the actual anatomical structures correspond one-to-one to the virtual reconstructed models. This 
method can not only reduce blind and excessive tissue separation but also avoids accidental structural injury, reduces intraoperative blood loss and improves the accuracy of the surgical technique. It may also explain why, in our study, the surgery time of the 3D-CTBA and 3D printing group was shorter than that of the general group, and the blood loss was significantly less than that of the general group. The risk of vascular injury caused by anatomical variation in the pulmonary segmental vessels during surgery is an important factor for conversion from thoracoscopic segmentectomy to thoracotomy or lobectomy. Therefore, fully understanding the individual anatomical variations of the pulmonary vessels through a preoperative 3D-CTBA and $3 \mathrm{D}$ printing model are important for operative safety. In our study, two patients were converted to thoracotomy or lobectomy due to bleeding of vascular injury or pulmonary segmental veins were mistakenly cut off in the general group, while no patient in the $3 \mathrm{D}-\mathrm{CTBA}$ and $3 \mathrm{D}$ printing group was converted to lobectomy or thoracotomy.

The advantage of preoperative 3D-CTBA is also reflected in the accurate localization of nodules, especially for multiple and segmental nodules (14). Currently, there are various methods of pulmonary nodule localization, such as ZT glue, CT-guided hook wires, spring coils, or methylene blue staining, which can effectively locate pulmonary nodules before surgery. For pulmonary wedge resection, these methods are simple and practical. However, the above-mentioned methods cannot completely solve the problem of accurate segmental attribution of the nodules, especially when the nodules are located at the edge of adjacent segments and it is difficult to locate the nodules by conventional two-dimensional CT images. Preoperative three-dimensional reconstruction can not only avoid the damage caused by puncture positioning but also can be accurately assigned to the subsegmental of the nodules. Because of the ability to accurately locate the nodule in the pulmonary subsegment, it is possible to determine whether a segmentectomy or a subsegmentectomy needs to be performed to ensure an adequate surgical margin and to preserve the intersubsegmental vein (15-18).

The 3D-CTBA and 3D printing model also displays some other advantages as follows: (I) the model can help surgeons make preoperative plans faster and better, reducing the difficulty of surgery, and thus shortening the learning curve for thoracic surgeons; (II) the application of a 3D-CTBA and 3D printing model can also promote doctorpatient communication. We routinely explain the operation plan and surgery-related risks to the family members of patients undergoing segmentectomy. Using this method, the family members of patients are better able to easily understand and accept the operation plan, which in turn reduces their preoperative anxiety; (III) 3D models are also very helpful for clinical teaching because they increase the trainees' three-dimensional perception and tactile feedback; (IV) $3 \mathrm{D}$ reconstruction can also accurately reflect the shape of lung lesions and the relationship between the peripheral vascular tissues and can help in differentiating benign and malignant pulmonary nodules.

In summary, 3D-CTBA has significant advantages in identifying anatomical variations and locating nodules. The $3 \mathrm{D}$ printing model can increase $3 \mathrm{D}$ perception and provide a good sense of space and tactile feedback. 3D-CTBA combined with a $3 \mathrm{D}$ printing model can improve the safety of segmentectomy or subsegmentectomy, and it has become an important auxiliary tool for thoracic surgeons.

\section{Acknowledgments}

Funding: This study was funded by the Shaoxing Science and Technology Project (No. 2020A13059).

\section{Footnote}

Reporting Checklist: The authors have completed the MDAR reporting checklist. Available at http://dx.doi.org/10.21037/ jtd-21-16

Data Sharing Statement: Available at http://dx.doi. org/10.21037/jtd-21-16

Conflicts of Interest: All authors have completed the ICMJE uniform disclosure form (available at http://dx.doi. org/10.21037/jtd-21-16). The authors have no conflicts of interest to declare.

Ethical Statement: The authors are accountable for all aspects of the work in ensuring that questions related to the accuracy or integrity of any part of the work are appropriately investigated and resolved. All procedures performed in this study involving human participants were in accordance with the Declaration of Helsinki (as revised in 2013). The study was approved by the Institutional Review Board of the Affiliated Hospital of Shaoxing University, and written informed consent was obtained from patients prior to surgery.

Open Access Statement: This is an Open Access article 
distributed in accordance with the Creative Commons Attribution-NonCommercial-NoDerivs 4.0 International License (CC BY-NC-ND 4.0), which permits the noncommercial replication and distribution of the article with the strict proviso that no changes or edits are made and the original work is properly cited (including links to both the formal publication through the relevant DOI and the license). See: https://creativecommons.org/licenses/by-nc-nd/4.0/.

\section{References}

1. Zhong C, Fang W, Mao T, et al. Comparison of thoracoscopic segmentectomy and thoracoscopic lobectomy for small-sized stage IA lung cancer. Ann Thorac Surg 2012;94:362-7.

2. Kodama K, Higashiyama M, Okami J, et al. Oncologic outcomes of segmentectomy versus lobectomy for clinical T1a N0 M0 non-small cell lung cancer. Ann Thorac Surg 2016;101:504-11.

3. Zhao ZR, Situ DR, Lau RWH, et al. Comparison of segmentectomy and lobectomy in stage IA adenocarcinomas J Thorac Oncol 2017;12:890-6.

4. Ginsberg RJ, Rubinstein LV. Randomized trial of lobectomy versus limited resection for T1 N0 non-small cell lung cancer. Lung Cancer Study Group. Ann Thorac Surg 1995;60:615-22; discussion 622-3.

5. Li F, Zhao Y, Yuan L, et al. Oncologic outcomes of segmentectomy vs lobectomy in pathologic stage IA $(\leq 2$ $\mathrm{cm}$ ) invasive lung adenocarcinoma: A population-based study. J Surg Oncol 2020;121:1132-9.

6. Chan EG, Chan PG, Mazur SN, et al. Outcomes with segmentectomy versus lobectomy in patients with clinical T1cN0M0 non-small cell lung cancer. J Thorac Cardiovasc Surg 2020. [Epub ahead of print]. doi: 10.1016/ j.jtcvs.2020.03.041.

7. Razi SS, Nguyen D, Villamizar N. Lobectomy does not confer survival advantage over segmentectomy for nonsmall cell lung cancer with unsuspected nodal disease. J Thorac Cardiovasc Surg 2020;159:2469-83.

8. Aokage K, Yoshida J, Hishida T, et al. Limited resection for early-stage non-small cell lung cancer as function preserving radical surgery: a review. Jpn J Clin Oncol 2017;47:7-11.

9. Qiu B, Ji Y, He HY, et al. Three-dimensional reconstruction/personalized three dimensional printed model for thoracoscopic anatomical partial-lobectomy in stage I lung cancer: a retrospective study. Transl Lung Cancer Res 2020;9:1235-46.
10. Liu X, Zhao Y, Xuan Y, et al. Three-dimensional printing in the preoperative planning of thoracoscopic pulmonary segmentectomy. Transl Lung Cancer Res 2019;8:929-37.

11. Zhang M, Liu D, Wu WB, et al. Preoperative 3D-CT bronchography and angiography facilitates single-direction uniportal thoracoscopic anatomic lobectomy. Ann Transl Med 2019;7:526.

12. Wu WB, Xia Y, Pan XL, et al. Three-dimensional navigation-guided thoracoscopic combined subsegmentectomy for intersegmental pulmonary nodules. Thoracic Cancer 2019;10:41-6.

13. She XW, Gu YB, Xu C, et al. Three-dimensional(3D)computed tomography bronchography and angiography combined with 3D-video-assisted thoracic surgery (VATS) versus conventional 2D-VATS anatomic pulmonary segmentectomy for the treatment of non-small cell lung cancer. Thoracic Cancer 2018;9:305-9.

14. Kato H, Oizumi H, Suzuki J, et al. Thoracoscopic anatomical lung segmentectomy using $3 \mathrm{D}$ computed tomography simulation without tumour markings for nonpalpable and non-visualized small lung nodules. Interact Cardiovasc Thorac Surg 2017;25:434-41.

15. Iwano. S. Planning video-assisted thoracic surgery segmentectomy using three dimensional computed tomography angiography and bronchography with a virtual safety margin. J Vis Surg 2017;3:82.

16. Shimizu K, Nakano T, Kamiyoshihara M, et al. Segmentectomy guided by three-dimensional computed tomography angiography and bronchography. Interact Cardiovasc Thorac Surg 2012;15:194-6.

17. Zabaleta J, Aguinagalde B, Lopez I, et al. Creation of a multidisciplinary and multicenter study group for the use of $3 \mathrm{D}$ printing in general thoracic surgery: lessons learned in our first year experience. Med Devices (Auckl) 2019;12:143-9.

18. Chan EG, Landreneau JR, Schuchert MJ, et al. Preoperative (3-dimensional) computed tomography lung reconstruction before anatomic segmentectomy or lobectomy for stage I non-small cell lung cancer. J Thorac Cardiovasc Surg 2015;150:523-8.

Cite this article as: $\mathrm{Hu} \mathrm{W}$, Zhang $\mathrm{K}$, Han $\mathrm{X}$, Zhao J, Wang G, Yuan S, He B. Three-dimensional computed tomography angiography and bronchography combined with three-dimensional printing for thoracoscopic pulmonary segmentectomy in stage IA non-small cell lung cancer. J Thorac Dis 2021;13(2):1187-1195. doi: 10.21037/jtd-21-16 